our globe, and the conclusion arrived at was, to "grant the first rank to the internal, volcanic, or cataclysmic agencies, since, had it not been for their operations, our globe would still have re mained a comparatively smooth sphere, surrounded by its exterral envelope of water, with no visible land for the rivers to traverse or the rain and ice to disintegrate and wear away," \&c.

In order that your numerous scientific public may not be led to judge of the lecture by this outline, I trust to your good will in asking you to insert these remarks in your next number.

II, York Place, Portman Square, Aug. 22 DAVID FORBES

\section{A Vivid Mirage}

THE illusion known as the mirage is, I believe, not unfrequently observed in the British Isles; but the vividness with which it was displayed on the present occasion will, I trust, be a sufficient apology for troubling you with this letter.

The land bordering the River Nene is protected by banks of from twelve to fifteen feet in height, enclosing a space called the "Wash," which receives the flood waters. It was from one of these banks that the appearance in question was observed, nothing unusual being seen from the level of the fen. I may mention that the Wash at this season is as dry as any other portion of the land.

The day (August I2) was hot, the sky cloudless, and a strong N.E. wind was blowing. About eleven o'clock the phenomenon was first noticed. To the eastwarl a dark line of trees, some eight miles distant, stood out in bold relief against the clear sky, and in front of this a shining line of silvery brightness was seen, which gradually widened until about twelve o'clock it presented the appearance of a broad expanse of water, ruffed into waves on its near side, but perfectly calm and clear toward the horizon where the line of trees was beautifully reflected on its surface. As I had been approaching the scene all this time, the expanding of the lake appeared perfectily natural, and I could scarcely heip thinking the river must have overflowed during the night and drowned the "Wash." This, of course, I knew to be quite out of the question, but the semblance was so perfect that it required an effort to believe that it was but an illusion. Its shores were clearly defined, little bays dimpled it, tiny headlands jutted out from it, and the waves were seen rising and falling with life-like exactitude. The whole appeared quite stationary, and as I approached the spot it gradually faded away, until nothing but a thin bine haze beneath the trees remained, and this at length dissolved.

On looking behind me (i.e. westward) another mirage seemed forming, which increased in apparent extent as I went farther from it. In this case the illusion was, if possible, more perfect than in the last, and the comparatively high land of Whittlesea rose like an island from the shining sea. Vehicles passing along the road seemed floating on its surface, their dark drawn-out reflections showing vividly against the sun-lit water, which, in this instance, was quite calm. How long this illusion lasted I know not, but when, about two o'clock, I quitted the bank it was still very distinct.

The dead level of the fen, and the bright sunlight falling upon the parched land, from which the heated air rose tremulous as from a hot plate, render this district peculiarly favourable to the production of such effects. For the accuracy with which the appearance of water was simulated it was quite equal to any mirage I have witnessed on the African deserts.

SyDNEY B. J. SKERTChly

Geological Survey, Whittlesea, Cambridge, Aug. 12

\section{Mirages made Easy}

THE very interesting account of a mirage in this week's NaTuR induces me to send a few observations. The mirage phenomenon is by no means so uncommon in England as many think. Three or four summers ago, on a strip of sand three miles long at Morcambe Bay, I was able to see one almost every hot day, by simply stooping until my eyes were about a yard above the ground. The further part of the sand then appeared as a lake of water, with objects reflected, \&c. The nearer edge of this lake receder as the eyes were raised, the whole soon pecoming invisible. I saw the same effects last summer off the Holderness Coast, but again only by stooping. At Cambridse I have lately seen a very good lateral mirage, by looking closely along the surface of a wall fifty yards in length, which had been exposed for some hours to a western sum. Objects near the further end of the wall were distinctly reflected.

Trin. Coll. Cambridge, Aug. 4

\section{Science and the Government}

THE announcement in your last number of a rumour that the Government is about to withdraw its promise of aid to the Total Eclipse Expedition, seems to bring to a climax the relations of the Government towards science. We can hardly forget that one of the prominent members of the present ministry, and the one considered to have the special control over the spending of the public funds, is member for the University of London. Mr. Lowe was sent to Parliament, irrespective of party considerations, as the representative of a body which thinks it has some claim to a leading place among the scientific institutions of our country. Is it not worth while to consider whether the views of the graduates of London University are represented in the present attitude of the ministry, and whether some representation might not be made to the Government, through the Chancellor of the Exchequer, of the manner in which the present relations between the Government and science are regarded by his constituents?

3, Park Village East, Aug. 20 Alfred W. Bennett

\section{AROMATYC GLYCOL*}

$A$ LL chemists recollect the profound impression caused $\mathrm{A}^{\mathrm{L}}$ by the discovery of glycol in 1855 by $\mathrm{M}$. Wurtz. Up to that time the bodies which were recognised as belonging to the group "alcohol" only included what we now call monatomic alcohols (common alcohol and its analogues), and M. Berthelot hesitated before renturing upon declaring glycerine a triatomic alcohol-an opinion to which Gerhardt never entirely adhered.

M. Wurtz showed that besides ordinary or monatomic alcohols, there are others which, when submitted to certain reagents where ordinary alcohols furnish only one, produce two derivatives. To these substances he gave the name of diatomic alcohols or glycols, and recognised that to each monatomicalcohol belonged a corresponding glycol, which only differed from it by the addition of an atom of oxygen. This new view became rapidly extended. It was admitted that belonging to each glycol there was, or might be, a triatomic alcohol or glycerine; that to each glycerine there might be a corresponding tetratomic alcohol, and so on; these alcohols only differing from one another by the number of atoms of oxygen which they contained, the number being always denoted by the atomicity of the alcohol.

Shortly before M. Wurtz's discovery of glycol, Signor Canizzaro, now Professor at the University of Palermo, but then Professor at Genoa, had announced the discovery of a new alcohol, which he called benzylic alcohol, having to benzoic acid the same relation as vinous alcohol has to acetic acid. He had obtained this substance by the action of potash in alcoholic solution, on the essence of bitter almonds.

Some time later the same chemist discovered that this alcohol might be equally obtained by means of toluol (toluène). The method he employed was to subject chlorinated toluol to the action of acetate of potash; and finally to decompose the acetate of benzyl thus obtained by means of potash.

This benzylic alcohol was the starting point of a new series of alcohols known as aromatic monatomic alcohols, and in fact soon afterwards cumylic alcohol was obtained, and Signor Canizzaro himself shortly afterwards published his discovery of tolylic alcohol.

It should, however, be observed that the process by means of which Signor Canizzaro had obtained his benzylic alcohol from toluol, succeeded ill with the homo-

\footnotetext{
* "Sur un Glycol Aromatique." Par M, Edouard Grimanx.
} 
logues of this hydro-carbon, and gave, even with tolucl, but small quantities of the alcohol.

Herr Beilstein, however, took up the matter at the point where others had abandoned it. He showed that when chlorine is made to act upon heated aromatic hyclrocarbons, there is obtained a chloro-derivative, easily acted upon by acetate of silver or of potassium, and consequentiy admirably fitted to furnish the corresponding alcohol; whilst on the other hand when used cold, a very stable chloro-substitution compound is formed of the same composition, but furnishing no alcohol.

From this moment no difficulty remained in completing the series of the aromatic monatomic alcohols; but up to this time no diatomic alcohol, or aromatic glycol was known. It was, however, evident that the preparation of such compounds was possible, inasmuch as the knowledge already existed of such acids as formobenzoilic acid, and terephtalic acid, which bore the same relation to these unknown bodies as glycolic and oxalic acids to ordinary glycol.

M. Edouard Grimaux, after much long and careful investigation, has at length filled up the blank by the discovery of tolylenic or xylenic glycol, that is to say a glycol which presents in its composition xylol to which two atoms of oxygen are added without substitution.

To obtain tolyl-glycol M. Grimaux takes the xylol of commerce which he raises to the boiling point in a retort, surmounted by an inverted refrigerator, from which the condensed vapours flow back into the retort, the tubulure of which is closed by a cork, into which a funnel with a tap is inserted. He then introduces drop by drop into the boiling hydro-carbon half its weight of bromine. The bromine is at once absorbed with the production of hydro: bromic acid, and on cooling a black crystalline mass is obtained which is first washed with ether, and its purification completed by crystallisation from chloroform. This substance is a bibromo-xylol casily acted on by re-agents.

Treated at a temperature of $150^{\circ}$, or in a closed vessel with an alcaholic solution of acetate of sodium, the bromoproduct gives rise by double decomposition to bromide of sodium and to diacetate of tolylene, which takes the form of beautiful colouriess crystals.

Subjected in its turn to the action of thirty times its weight of water at a temperature of $170^{\circ}$ or $180^{\circ}$ in a closed vessel, this acetate of tolylene is separated into acetic acid and tolylenic glycol, which, on the opening of the tube and by the evaporation of the liquid, is deposited in needle-shaped crystals.

The formation of tolyl-glycol may be represented by the following equations :-

$$
\begin{gathered}
\left.\mathrm{C}_{6} \mathrm{H}_{4}<\underset{\mathrm{CH}_{3}}{\mathrm{CH}_{3}}+{ }_{2}\left\{\begin{array}{l}
\mathrm{Br} \\
\mathrm{Br}
\end{array}\right\}\right)= \\
\text { Xylol. } \\
\text { Bramine. }
\end{gathered}
$$

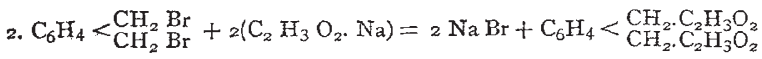

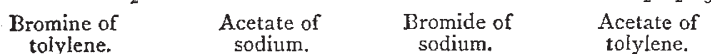

3. $\left.\mathrm{C}_{6} \mathrm{H}_{4}<\underset{\mathrm{CH}_{2}}{\mathrm{CH}_{2} \cdot \mathrm{C}_{2} \mathrm{H}_{3} \mathrm{O}_{2}+\mathrm{H}_{2} \mathrm{O}_{2}}(\stackrel{\mathrm{H}}{\mathrm{H}}\} \mathrm{O}\right)=2\left\langle\mathrm{C}_{2} \mathrm{H}_{3} \mathrm{O}_{2} \mathrm{H}\right\rangle+\cdots \mathrm{C}_{5} \mathrm{H}_{4}<\underset{\mathrm{C} \mathrm{CH}_{2} \mathrm{OH}}{\mathrm{C}}$

$$
\begin{aligned}
& \text { Acetate of } \\
& \text { Water. Acetic acid. Tolyl glycol. }
\end{aligned}
$$
Acetate of
toluol.

The path is now open, and nothing will be easier than, by following the method proposed by $M$. Grimaux, to prepare all the aromatic glycols, of which the hydrocarburets are already known to us.

ALFRED NAQUET

\section{SPECTROSCOPIC NOTES *}

SPECTRUM OF A SOlaR SPOT, APRIL 9, r870, P.M. FXAMINED the spectrum of a large group of spots E little north and east of the sun's centre.

The nucleus of the most southerly member of the group * Communicated by the Author. reversed the $C$ line finely, turning it into a conspicuous bright line for about $20^{\prime \prime}$ of its length, without any distortion, however, such as is commion upon this (dark) line in the neighbourhood of spots.

F was also reversed, but rather faintly.

$\mathrm{D}_{3}$ could not be made out at all in the nucleus spectrum ; neither were 2796 (the numbers refer to Kirchhoff's map) nor $\hbar$ reversed. I thought, however, that they were somewhat thimned.

The reversal of $C$ and $F$ continued through the whole afternoon.

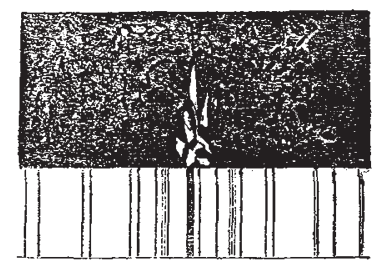

On the other hand, many of the dark lines were widened and deepened in this nucleus spectrum in the manner which the description and figures of Mr. Lockyer have made familiar. Many also vere unaffected. Among these were notably $a, B, E, 1474$, the four lines of $b, 169 \mathrm{r}$ and $\mathrm{G}$.

The two sodium lines $\mathrm{D}_{x}$ and $\mathrm{D}_{2}$, and $88_{5}$ ( $F \mathrm{C}$ ) were distinctly, but not greatly, widened.

The effect was most marked upon the following : 864 (Ca), 877 ( $\mathrm{Fe}$ ?), 885 (Ca), 895 ( $\mathrm{Ca}$ and $\mathrm{Li}$ ), 1580 (Ti), I 599 (Ti), 1627 (Ca), and 1629 (Ti). I have marked 877 doubtful, because there lies very near it a line whose origin is unknown, and I am not sure to which of the two the thickening was due. The Titanium lines are identified as such by reference to Angström's Atlas. I was greatly surprised at the prominence they assume in the spot-spectrum, as they are inconspicuous in the normal spectrum; and a similar remark applies to the calcium lines.

I do not intend to convey the idea that the lines mentioned were the only ones that were much deepened ; there were many others, mostly faint, affected to nearly the same degree, but I had not time to identify them.

There was at the same time an exceedingly brilliant protuberance on the south-west limb of the sun (position angle $230^{\circ}$ ), near but not over, a large spot which was just passing off. At the base of this prominence, which was shaped like a double ostrich plume, the $C$ line was intensely brilliant, so that the slit could be opened to its whole width in studying the form above described, but it was not, so far as I could see, in the least distorted, On the other hand, the F line, also very brilliant, was shattered all to pieces, so that at its base it was three or four times. as wide as ordinary, and several portions of it were entirely detached from the rest. The figure, without pretending to exact accuracy, and for the sake of distinctness a little exaggerated, gives a fair idea of the nature and extent of the "shattering" alluded to.

Since the C line was not similariy affected it is hardly possible to attribute this breaking up of $F$ to cyclonic motions in the gas from which the light emanates, and it becomes very difficult to imagine a cause that can thus disturb a single line of the spectrum by itself. Possibly this appearance may be the result of local absorptions acting upon a line greatly widened by increase of pressure or temperature.

It continued unchanged for more than half an hour, and until the sun passed out of sight behind a building. The observations were made with the 5 prism spectroscope.

Hanover, New Hampshire, May 20 c. A. YounG 